\title{
Nesfatina-1: una nueva hormona anorexigénica
}

\author{
Alejandra López-Gómez ${ }^{1}$ y Claudia Martínez-Cordero ${ }^{2^{*}}$ \\ ${ }^{1}$ Universidad Iberoamericana León; ${ }^{2}$ Hospital Regional de Alta Especialidad del Bajío. León, Guanajuato, México
}

\section{RESUMEN}

La nesfatina-1 (N-1) es una hormona anorexigénica de reciente descubrimiento que regula la homeostasis energética y actúa a nivel central para disminuir la ingesta de alimentos; y por otro lado, inhibe el vaciamiento gástrico y reduce la motilidad gastrointestinal. La N-1 es un péptido neuroendocrino que se secreta por diversas neuronas del sistema nervioso central (SNC) y tejidos periféricos como el tejido adiposo, páncreas y tracto gastrointestinal, por lo que actúa a nivel central y periférico para contribuir principalmente al balance energético. La N-1 es una hormona con propiedades antihiperglucémicas que estimula la secreción de insulina y promueve la captación de glucosa periférica. Actualmente se investigan nuevas funciones de la N-1 en el organismo.

Palabras clave: Nesfatina-1. Hormona anorexigénica. Péptido neuroendocrino. Balance energético.

\section{ABSTRACT}

Nesfatin-1 ( $\mathrm{N}-1)$ is an anorexigenic hormone recently discovered that regulates energy homeostasis, and acts at the central level to decrease food intake; on the other hand, it inhibits gastric emptying and reduces gastrointestinal motility. $\mathrm{N}-1$ is a neuroendocrine peptide that is secreted by several central nervous system neurons and by peripheral tissues such as adipose tissue, pancreas and gastrointestinal tract, thus acting at the central and peripheral levels to contribute mainly to the energy balance. $\mathrm{N}-1$ is a hormone with anti-hyperglycemic properties that stimulates the secretion of insulin and promotes the uptake of peripheral glucose. At present, research is finding new functions of $\mathrm{N}-1$ in the body. (Hosp Med Clin Manag. 2018;11:130-6)

Corresponding author: Claudia Martínez-Cordero, claudiamartinezcordero@ hotmail.com

Key words: Nesfatin-1. Anorexigenic hormone. Neuroendocrine peptide. Energy balance.

\section{INTRODUCCIÓN}

La $\mathrm{N}$-1 es un péptido neuroendocrino de 82 aminoácidos regulador de la saciedad ${ }^{1}$. Oh-I, et al. (2006) descubrieron la $\mathrm{N}-1$ en el núcleo hipotalámico del control del apetito en ratas causando anorexia en estos animales ${ }^{2}$. El nucleobindin 2 (NUCB2) es el precursor de la $\mathrm{N}$-1; ambos se expresan y secretan en el SNC y tejidos periféricos, se involucran con el metabolismo ${ }^{3}$ y actúan en varios procesos homeostáticos ${ }^{4}$. La N-1 se colocaliza con diversos

\section{Correspondencia:}

*Claudia Martínez-Cordero

Hospital Regional de Alta Especialidad del Bajío

Blvd. Milenio 130

Col. San Carlos La Roncha

Date of reception: 21-12-2017

C.P. 37660, León, Guanajuato, México

Date of acceptance: 12-09-2018

E-mail: claudiamartinezcordero@ hotmail.com

Doi: 10.24875/HMCM.18000095 
neurotransmisores y péptidos involucrados en la modulación del metabolismo, homeostasis energética e ingesta de alimentos ${ }^{3}$, como la urocortina-1, la hormona concentradora de melanina $(\mathrm{MCH})$, la proopiomelanocortina (POMC), el péptido relacionado a la cocaína y anfetamina, la hormona estimuladora de melanocitos $\alpha(\mathrm{MSH}-\alpha)$, la vasopresina, el neuropéptido Y (NPY), la oxitocina, la hormona liberadora de la hormona de crecimiento, el factor liberador de corticotropina (CRF), la hormona liberadora de tirotropina $(\mathrm{TRH})$, la somatostatina y la serotonina ${ }^{5}$.

En los tejidos periféricos, la N-1 se localiza en el tejido adiposo, mucosa gástrica, páncreas, testículos ${ }^{6}$, corazón ${ }^{7}$, músculo esquelético, hígado, pulmón y riñón, entre otros sitios $^{8}$, y se colocaliza con la grelina en las células gástricas en ratas y en humanos, así como también con la insulina en los islotes pancreáticos ${ }^{7}$. La amplia distribución periférica de NUCB2/N-1 influye en la alimentación y otras acciones metabólicas ${ }^{5}$, las cuales dependen de su sitio de localización. Se desconoce cuál es el receptor de la $\mathrm{N}-1$, pero se considera que es el receptor acoplado a proteínas $\mathrm{G}$ que está distribuido en los órganos periféricos, como el estómago, intestino delgado, órganos endocrinos, tejido adiposo visceral, corazón, músculo esquelético, hígado, pulmón y riñón en ratas ${ }^{7}$. Este péptido se mide en plasma y se altera bajo diversas condiciones en los humanos ${ }^{3}$, ya que -entre otros factores- sus niveles dependen de la ingesta de alimentos (los niveles disminuyen durante el ayuno y se reestablecen después de ingerir alimentos) $)^{9}$. Por todo lo anterior, se considera que los niveles plasmáticos de $\mathrm{N}-1$ dependen del estado nutricional y de diversas circunstancias metabólicas ${ }^{10}$. No se ha establecido un rango normal de N-1 plasmática.

\section{LA NESFATINA-1 Y EL APETITO}

La regulación de la ingesta de alimentos se logra por señales endocrinas centrales y periféricas, las cuales actúan como anorexigénicas u orexigénicas ${ }^{11}$. La N-1 es un modulador anorexigénico que contribuye al control o disminución del peso corporal ${ }^{9}$, y su efecto anorexigénico se produce a nivel central, pero continúa investigándose si afecta también a nivel periférico. Cuando se descubrió la $\mathrm{N}-1$, se demostró que la inyección intracerebroventricular (icv.) de NUCB2 disminuye el apetito y peso corporal en ratas ${ }^{2}$. La disminución del apetito por la inyección icv. de $\mathrm{N}-1$ es dosis-dependiente, y la inyección de un anticuerpo neutralizante de $\mathrm{N}-1$ estimula el apetito $^{12}$. La acción anorexigénica de $\mathrm{N}-1$ se produce a las $2 \mathrm{~h}$ de su administración y su efecto dura $8 \mathrm{~h}^{5}$. Sin embargo, el mecanismo de acción de la N-1 aún no está claro, por lo que se presentan dos hipótesis: activación del sistema oxitocina-POMC-MSH- $\alpha$-melanocortina3/4 y aumento de la expresión de CRF y TRH, así como por la inhibición directa de sustancias orexigénicas ${ }^{6,13}$.

El primer mecanismo de la acción anorexigénica de la $\mathrm{N}-1$ se puede deber a la activación de una vía de señalización del receptor hipotalámico oxitocina-POMC-MSH$\alpha-M C 3 / 4^{14}$. La POMC es un anorexigénico escindido a varios péptidos, incluyendo la $\mathrm{MSH}-\alpha$, que es un ligando endógeno del receptor de $\mathrm{MC} 3 / 4^{15}$. Al bloquear el receptor $\mathrm{MSH}-\alpha \mathrm{MC} 3 / 4$ se bloquea el efecto anorexigénico de la $\mathrm{N}-1$ inyectada icv. Además, la inyección en el tercer ventrículo de $\mathrm{MSH}-\alpha$ aumenta la expresión del ARNm de NUCB2 en el núcleo paraventricular $(P V N)^{5}$. La inyección de $\mathrm{N}-1$ en el tercer ventrículo incrementa el flujo de calcio $\left(\mathrm{Ca}^{2+}\right)$ en las neuronas que contienen oxitocina, lo cual estimula su liberación y ocasiona un efecto anorexigéni$\mathrm{Co}^{16}$. Por otra parte, la inyección icv. de N-1 en ratas incrementa el contenido de CRF en el PVN disminuyendo la ingesta de alimentos. En cuanto a la TRH, ésta puede estar implicada en los efectos anorexigénicos de la $\mathrm{N}-1$, ya que la expresión de ARNm de TRH en el PVN se aumenta después de la inyección icv. de $\mathrm{N}-1$, y la inyección de anticuerpos de TRH en el tercer ventrículo inhibe la $\mathrm{N}-1$ en ratas ${ }^{5}$. El segundo mecanismo de la acción anorexigénica de $\mathrm{N}$-1 es por la inhibición directa de sustancias orexigénicas, como lo es el NPY. La administración de $\mathrm{N}-1$ en neuronas del núcleo arcuato (ARC) in vitro resulta en la hiperpolarización de neuronas de NPY, que probable promueva un efecto anorexigénico ${ }^{6,13}$.

La acción anorexigénica de la $\mathrm{N}-1$ a nivel periférico es menos consistente que a nivel cerebral, ya que se han presentado estudios contradictorios ${ }^{14,17}$. Sin embargo, Shimizu, et al. demostraron que la inyección intraperitoneal (ip.) de $\mathrm{N}-1$ disminuye la ingesta de alimentos de manera dosis-dependiente, y que su efecto se produce si se inyecta $\mathrm{N}-1$ de ratas o de humanos ${ }^{18}$. Se considera que el mecanismo por el cual la inyección periférica de N-1 actúa involucra la vía vagal aferente, ya que esta hormona activa el flujo de $\mathrm{Ca}^{2+}$ en los ganglios nodosos neuronales in vitro en ratones ${ }^{5}$. Los efectos anorexigénicos de esta hormona se consideran independientes de la señalización de la leptina, tanto a nivel central como periférico, ya que la $\mathrm{N}-1$ continúa inhibiendo la ingesta de alimentos en roedores con resistencia a la leptina cuando se inyecta de manera icv. o ip. ${ }^{5,18}$ (Tabla 1). 
Tabla 1. Estudios sobre los efectos de la N-1 en la ingesta de alimentos, composición corporal, tracto gastrointestinal y homeostasis de la glucosa

\begin{tabular}{|c|c|c|}
\hline Autor, año y referencia & Procedimiento & Resultados \\
\hline \multicolumn{3}{|l|}{ Ingesta de alimentos } \\
\hline Oh-I, et al. $(2006)^{2}$ & Inyección icv. de $\mathrm{N}-1$ en ratas & $\begin{array}{l}\text { Peso corporal y apetito disminuyen de forma dosis- } \\
\text { dependiente }\end{array}$ \\
\hline Stengel, et al. $(2013)^{5}$ & Inyección icv. de $\mathrm{N}-1$ en ratas & $\begin{array}{l}\text { Acción anorexigénica durante } 8 \mathrm{~h} \text { después de } 2 \text { h de la } \\
\text { administración }\end{array}$ \\
\hline Shimizu, et al. (2009) ${ }^{18}$ & Inyección ip. de N-1 en ratones & $\begin{array}{l}\text { Ingesta de alimentos disminuye de forma dosis- } \\
\text { dependiente }\end{array}$ \\
\hline Stengel, et al. $(2013)^{5}$ & $\begin{array}{l}\text { Inyección icv. de } \mathrm{N}-1 \text { en ratas deficientes de receptores } \\
\text { de leptina }\end{array}$ & $\begin{array}{l}\text { Ingesta de alimentos suprimida; efecto anorexigénico } \\
\text { independiente a la señalización de leptina }\end{array}$ \\
\hline Shimizu, et al. $(2009)^{18}$ & $\begin{array}{l}\text { Inyección ip. de } \mathrm{N}-1 \text { en ratones con o sin resistencia a } \\
\text { leptina }\end{array}$ & Señalización N-1 a nivel periférico independiente de leptina \\
\hline
\end{tabular}

\section{Composición corporal}

Ramanjaneya, et al. (2010) ${ }^{22}$ Tejido adiposo en roedores y humanos

Expresión NUCB2/N-1 aumenta durante la diferenciación de adipocitos

Zhang, et al. (2017) $)^{19} \quad \mathrm{~N}-1$ en tejido adiposo humano

$\mathrm{N}-1$ aumenta en proporción a la adiposidad (porcentaje de grasa)

Mirzaei, et al. (2014) $\quad$ N-1 en obesidad y obesidad mórbida

$\mathrm{N}-1$ se asocia positivamente con la grasa corporal, pero no con el IMC

St-Pierre, et al. $(2016)^{23} \quad$ N-1 en obesidad severa con y sin cirugía bariátrica

$\mathrm{N}-1$ se correlaciona positivamente con el IMC, peso y masa grasa en pacientes con cirugía bariátrica

Tan, et al. $(2011)^{26} \quad$ NUCB2/N-1 e IMC

NUCB2/N-1 se correlaciona positivamente con el IMC, masa grasa y peso corporal

Saldanha, et al. (2012) ${ }^{27} \quad$ Pacientes con hemodiálisis

$\mathrm{N}-1$ se correlaciona positivamente con el IMC y grasa corporal porcentual. El porcentaje de grasa determina los niveles de $\mathrm{N}-1$

Anik, et al. $(2014)^{28} \quad$ Niños con obesidad

$\mathrm{N}-1$ sin correlación con parámetros antropométricos y metabólicos

Ustabas, et al. $(2015)^{29} \quad$ Niños con bajo peso

Niveles de N-1 más bajos en comparación con niños sanos

\section{Colocalización con grelina y función gastrointestinal}

Stengel, et al. (2013) $31 \quad$ Expresión de NUCB2/N-1 en tejido gástrico de pacientes con cirugía bariátrica

$\mathrm{Xu}$, et al. $(2017)^{32}$

Stengel, et al. $(2013)^{5}$

Inyección de N-1 en LHA

Inyección icv. De N-1 en ratas y ratones

Gao, et al. $(2017)^{34}$

Gao, et al. (2017) 34

Ramesh, et al. $(2016)^{35}$

Inyección de N-1 en PVN y ARC

Inyección de N-1 en VMH

Localización de N-1 en células enteroendocrinas en ratones machos
N-1 y grelina se colocalizan en la mucosa gástrica. Las células inmunorreactivas de grelina disminuyen, mientras que las células que expresan NUCB2/N-1 aumentan conforme mayor es el IMC

Motilidad y secreción gástrica inhibidas

Vaciamiento gástrico y motilidad gastrointestinal disminuidas; estimulación vagal inhibida para secretar ácido gástrico

Motilidad gástrica inhibida

Ingesta de alimentos, motilidad gástrica, producción de ácido gástrico y vaciamiento gástrico inhibidos

NUCB2/N-1 se colocaliza con CCK y PYY en las células enteroendocrinas intestinales. N-1 estimula la expresión de CCK, e inhibe la expresión y secreción de PYY

\section{Localización y función en la homeostasis de la glucosa}

\begin{tabular}{|c|c|c|}
\hline Li, et al. $(2013)^{40}$ & Infusión periférica de N-1 en ratones machos & $\begin{array}{l}\text { Fosforilación de AKT y translocación de GLUT4 aumentan, } \\
\text { promoviendo la captación de glucosa }\end{array}$ \\
\hline González, et al. $(2011)^{36}$ & $\mathrm{~N}-1$ en células b pancreáticas de ratones & $\begin{array}{l}\text { Expresión de ARNm de preproinsulina incrementa, al igual } \\
\text { que aumenta la secreción de insulina de manera } \\
\text { dependiente a la dosis de } \mathrm{N}-1 \text { y concentración de glucosa }\end{array}$ \\
\hline Su, et al. $(2010)^{41}$ & $\begin{array}{l}\text { Inyección intravenosa de } \mathrm{N}-1 \text { en ratones db/db } \\
\text { hiperglucémicos }\end{array}$ & $\begin{array}{l}\text { Glucosa en niveles bajos por } 6 \mathrm{~h} \text { sin ninguna } \\
\text { administración adicional de insulina }\end{array}$ \\
\hline
\end{tabular}




\section{LA NESFATINA-1 Y LA COMPOSICIÓN CORPORAL}

El tejido adiposo se considera un reservorio energético y un órgano endocrino activo ${ }^{19}$ que, por medio de la liberación de adipocinas (p. ej., la $\mathrm{N}-1)^{3}$, actúa en la regulación del apetito, distribución de grasa, gasto energético, inflamación, homeostasis, función endotelial y otras funciones. A nivel periférico, las adipocinas regulan diferentes procesos biológicos en diversos órganos, como el hígado, el músculo, el corazón y el páncreas $^{20}$. La producción de NUCB2 y N-1 en el tejido adiposo blanco es regulado por el sistema nervioso simpático, y su liberación parece estar involucrada en la transmisión de la señal anorexigénica desde la periferia hacia el cerebro ${ }^{21}$.

El proceso de diferenciación y maduración del adipocito incrementa la expresión de adipocinas biológicamente activas (leptina, adiponectina y apelina), y se considera que la $\mathrm{N}-1$ sigue un patrón similar con un incremento significativo en su expresión durante el proceso de diferenciación de preadipocitos hacia adipocitos maduros, por lo que es posible que la NUCB2/N-1 tenga un papel en el desarrollo y diferenciación del adipocito, aunque el hecho de que su secreción aumente a medida que los adipocitos maduran también sugiere que el incremento de su expresión es un subproducto, más que una causa ${ }^{22}$. Asimismo, en experimentos in vitro se ha demostrado un aumento similar de marcadores de diferenciación de adipocitos de receptores activados por proliferadores de peroxisomas $\gamma$ (PPAR- $\gamma$ ) y expresión intracelular de N-1. Los PPAR- $\gamma$ pertenecen a la superfamilia de receptores nucleares del factor de transcripción activado por ligando, y tienen una vía interconectada en la producción de adipocinas, por lo que se considera que la $\mathrm{N}-1$ tiene un efecto regulador en la adipogénesis ${ }^{3}$.

Considerando la producción de N-1 por el tejido adiposo, la concentración de esta adipocina aumenta según la proporción de adiposidad en humanos, por lo que el porcentaje de grasa corporal determina los niveles de $\mathrm{N}-1$ circulantes $^{19}$; sin embargo, la asociación de N-1 con el porcentaje de grasa corporal, masa grasa e índice de masa corporal (IMC) podría llegar a una meseta conforme aumenta el grado de obesidad, ya que mientras que el tejido adiposo es la principal fuente de $\mathrm{N}-1$, la acumulación de grasa en la obesidad puede alterar la proporción central/periférica de esta adipocina, y subsecuentemente afectar a su correlación con el IMC y la masa grasa ${ }^{3}$. Se han demostrado correlaciones positivas, negativas o no correlación entre niveles circulantes de $\mathrm{N}-1$ y parámetros de composición corporal (principalmente IMC), y por lo tanto se sigue investigando ${ }^{9}$.

Mirzaei, et al. demostraron una asociación lineal entre la N-1 circulante y la concentración de PPAR-g en sujetos con obesidad y obesidad mórbida, así como un aumento de la concentración de $\mathrm{N}-1$ en sujetos con obesidad al incrementar la grasa corporal, pero sin demostrarse su correlación con el IMC ${ }^{3}$. St-Pierre, et al. demostraron que los niveles de $\mathrm{N}-1$ disminuyen en mujeres y hombres con obesidad severa que se someten a cirugía bariátrica, en comparación con controles con obesidad severa sin cirugía. En pacientes sometidos a cirugía bariátrica, los niveles de N-1 se correlacionan negativamente con parámetros de composición corporal (peso, masa grasa y masa libre de grasa) 5 días después de la cirugía, pero después de 12 meses las disminuciones de $\mathrm{N}-1$ se asocian con reducciones importantes en parámetros de composición corporal (IMC, peso y masa grasa) y de salud cardiometabólica (insulina en ayuno, glucosa en ayuno, HOMA-IR, colesterol total, LDL, apolipoproteína B, triglicérido y proteína C reactiva), lo cual sugiere que la $\mathrm{N}-1$ regula parámetros metabólicos del control de la composición corporal e inflamación, así como en la homeostasis de glucosa e insulina ${ }^{23}$.

Otro estudio en pacientes con obesidad severa que se sometieron a cirugía bariátrica demostró que el IMC postoperatorio a los tres y seis meses disminuyó significativamente en comparación con el preoperatorio, mientras que los niveles de $\mathrm{N}-1$ a los tres y seis meses postoperatorios fueron significativamente más altos que los preoperatorios ${ }^{24}$. Ozkan, et al. analizaron los cambios en los niveles de $\mathrm{N}-1$ de acuerdo al IMC, para lo cual dividieron a los participantes en cinco grupos de IMC (bajo-peso, normopeso, sobrepeso, obesidad y obesidad severa). Los autores demostraron que la N-1 aumentó conforme el IMC incrementó desde bajo-peso hasta sobrepeso, mientras que en obesidad volvió a disminuir y se incrementó nuevamente en el de obesidad severa. En consecuencia, no se observó correlación alguna entre los niveles de $\mathrm{N}-1$ y el IMC 25 .

Tan, et al. analizaron los niveles de NUCB2/N-1 en plasma y demostraron que, en sujetos con un IMC de 
16.2-38.1 kg/m², los niveles de NUCB2/N-1 plasmáticos se correlacionan de manera positiva con el IMC, masa grasa, edad, peso corporal, insulina plasmática y HOMA-IR, pero ninguna de estas variables es predictiva de los niveles de $\mathrm{N}-1 / \mathrm{NUCB}{ }^{26}$. Saldanha, et al. determinaron que la $\mathrm{N}-1$ se correlaciona positivamente con el IMC, la leptina y el porcentaje de grasa corporal en pacientes en hemodiálisis, así como que el porcentaje de grasa es el principal determinante de $\mathrm{N}-1$. Es posible que la eficiencia de la absorción de NUCB2/N-1 en el líquido cefalorraquídeo disminuya en personas con obesidad debido a la saturación de los transportadores $^{27}$. La N-1 no se correlacionó con parámetros antropométricos ni metabólicos en niños con obesidad ${ }^{28}$, pero se observaron niveles bajos de $\mathrm{N}-1$ en niños con bajo peso, en comparación con niños sanos. En consecuencia, se hipotetiza que niveles bajos de $\mathrm{N}-1$ podrían ser una respuesta que protege al organismo contra un peor estado nutricional ${ }^{29}$ (Tabla 1).

\section{LA NESFATINA-1 EN EL TRACTO GASTROINTESTINAL}

Diversas células neuroendocrinas se localizan en la mucosa del tracto gastrointestinal, las cuales se establecen como principales reguladoras de la ingesta de alimentos y funciones intestinales debido a la producción y liberación de hormonas peptídicas. El estómago contiene distintas células, como las liberadoras de gastrina, productoras de somatostatina, histamina y serotonina. Un quinto tipo de células endocrinas se ha descrito en la mucosa gástrica oxíntica, las llamadas P/D1 en humanos y tipo X/A en ratas, las cuales representan el 20-30\% de las células gástricas, y se estableció su función al descubrir la expresión de la grelina ${ }^{30}$.

La grelina es una hormona localizada en el estómago e intestino que regula varios procesos fisiológicos, particularmente del metabolismo y balance energético ${ }^{11}$. La grelina es la única hormona conocida que se produce periféricamente y actúa de manera central estimulando la ingesta de alimentos en los humanos (hormona orexigénica). Los niveles plasmáticos de esta hormona disminuyen en la obesidad y aumentan en la anorexia, lo que indica ser un mecanismo regulador de ingesta de alimentos ${ }^{5}$. La principal fuente de grelina es la célula gástrica endocrina $X / A$ en ratas, donde posteriormente se identificó la $\mathrm{N}-1^{30}$, lo que indica que ambas hormonas se colocalizan en las células gástricas ${ }^{5}$. La expresión de la prohormona convertasa1/3 en las células tipo X/A también apoya la coexpresión de grelina y $\mathrm{N}-1$, ya que esta enzima se requiere para madurar ambas hormonas peptídicas ${ }^{30}$.

La expresión de $\mathrm{N}-1$ en la mucosa gástrica es 20 veces mayor que su expresión a nivel cerebral6, lo que convierte al estómago en una fuente importante de $\mathrm{N}-1$, asumiendo que la célula X/A es estimuladora e inhibidora del consumo de alimentos, es decir, doble reguladora de la ingesta ${ }^{30}$. Stengel, et al. investigaron la expresión de NUCB2/N-1 en las células gástricas junto con grelina en tejido gástrico recolectado de pacientes con cirugía bariátrica, y observaron que la grelina y la $\mathrm{N}-1$ se colocalizan ampliamente en la mucosa gástrica humana (78\%), así como también observaron que el número de células inmunorreactivas de grelina disminuye, mientras que las células NUCB2/N-1 aumentan, conforme es mayor el IMC, lo que puede ser un mecanismo fisiológico adaptativo para prevenir la sobrealimentación bajo condiciones de IMC alto ${ }^{31}$.

La $\mathrm{N}-1$ regula la motilidad gástrica ${ }^{32}$, ya que se ha demostrado que la inyección icv. de N-1 en roedores disminuye el vaciamiento y la motilidad gastrointestinal, así como la secreción de ácido gástrico ${ }^{5}$. La N-1 está presente en las neuronas del núcleo del tracto solitario que se activan por distensión gástrica ${ }^{33}$. La $\mathrm{MCH}$, antagonista de $\mathrm{MSH}-\mathrm{a}$, se asocia con muchas funciones cerebrales, como ingesta de alimentos, homeostasis energética, respuesta al estrés y ansiedad. El área hipotalámica lateral (LHA) es una región del cerebro que regula múltiples procesos, como la alimentación y la función anatómica, y la administración en esta área de $\mathrm{N}-1$ cambia la actividad neuronal de la $\mathrm{MCH}$ que regula la distensión gástrica, lo que inhibe la motilidad y la secreción gástrica, mientras que la $\mathrm{MCH}$ en el LHA promueve la contracción y la secreción gástrica ${ }^{32}$. Asimismo, la inyección de N-1 en el PVN y ARC tiene un efecto inhibitorio en la motilidad gástrica, así como la inyección de $\mathrm{N}-1$ en el núcleo hipotalámico ventromedial (VMH) inhibe la ingesta de alimentos, la producción de ácido gástrico, la motilidad y el vaciamiento gástrico ${ }^{34}$.

La N-1 se colocaliza también con colecistoquinina (CCK) y péptido Y (PYY) en el intestino largo y corto de ratones machos, y la $\mathrm{N}$-1 estimula la CCK mientras inhibe la expresión y liberación de PYY. La CCK se secreta en respuesta a ingerir alimentos y es un potente 
anorexigénico que inhibe el vaciamiento gástrico, mientras que el PYY se secreta en respuesta a un alimento rico en proteínas, y su forma endógena, PYY3-36, reduce la ingesta de alimentos y el aumento de peso en roedores $^{35}$ (Tabla 1).

\section{LA NESFATINA-1 Y LA HOMEOSTASIS DE LA GLUCOSA}

La $\mathrm{N}-1$ es un péptido insulinotrópico que se colocaliza con la insulina en las células b pancreáticas y se altera en la diabetes, estimulando la secreción de insulina e incrementando la expresión de ARNm de preproinsulina y el flujo de $\mathrm{Ca}^{2+}$. La N-1 también se libera de manera independiente a la concentración de glucosa, incrementándose hasta cuatro veces en glucosas elevadas, por lo que podría regular la homeostasis de la gluco$\mathrm{sa}^{36}$. Esta hormona se correlaciona positivamente con la expresión del gen y la capacidad de secreción de insulina ${ }^{37}$, además de mejorar la acción de la insulina, ya que disminuye los niveles de glucosa en la sangre al mejorar la sensibilidad a la insulina a nivel periférico y hepático a través de la promoción de captación de glucosa periférica y disminución de la gluconeogénesis en ratones hiperglucémicos ${ }^{38,39}$. La infusión periférica de N-1 incrementa la fosforilación de la cinasa AKT en el músculo esquelético, hígado y tejido adiposo; por lo tanto, capta la glucosa aumentando la translocación del transportador de glucosa GLUT4 hacia la membrana celular ${ }^{40}$.

El efecto antihiperglucémico de la $\mathrm{N}-1$ es periférico y depende del tiempo, dosis e insulina, así como la administración icv. de $\mathrm{N}-1$ en ratones inhibe la ingesta de alimentos, pero no afecta a los niveles elevados de glucosa, por lo que su efecto anorexigénico es independiente al antihiperglucémico ${ }^{6}$. En ratones $\mathrm{db} / \mathrm{db}$ hiperglucémicos con diabetes mellitus, una inyección intravenosa de $10 \mathrm{nmol}$ de $\mathrm{N}-1$ mantiene bajos los niveles de glucosa en la sangre por $6 \mathrm{~h}$ sin ninguna administración adicional de insulina. La vida media de la $\mathrm{N}-1$ se ha reportado en 9-10 min, y sus efectos antihiperglucémicos duran horas en ratones hiperglucémicos $\mathrm{db} / \mathrm{db}$, lo que sugiere que su efecto intracelular sería duradero y afectaría de manera importante el metabolismo de la glucosa ${ }^{41}$. Li, et al. analizaron los efectos de la N-1 periférica en la homeostasis de la glucosa en ratones alimentados con dos tipos de dieta: normal y alta en grasa. Mediante la prueba de tolerancia oral a la glucosa y la prueba de tolerancia a la insulina, demostraron que los ratones tratados con $\mathrm{N}-1$ mostraban un mejor metabolismo de la glucosa, tanto los ratones con dieta normal como los que seguían una dieta alta en grasa ${ }^{40}$.

Al examinar los niveles de $\mathrm{N}-1$ en pacientes con glucosa alterada en ayuno (IFG), tolerancia alterada de glucosa (IGT), diabetes mellitus de tipo 2 (DM2), síndrome metabólico y sanos (control), se mostró que los niveles de $\mathrm{N}-1$ más bajos se presentaron en el grupo con IFG, seguido por el de IGT y el de control. Por el contrario, los pacientes con DM2 y síndrome metabólico presentaron niveles de $\mathrm{N}-1$ significativamente más bajos, en comparación con el grupo de control, lo que puede ser una de las razones de la hiperfagia diabética. Además, se encontró diferencia en la resistencia a la insulina entre el grupo con síndrome metabólico y DM2, y con IFG e IGT. Considerando lo anterior, la resistencia a la insulina y la alteración en la secreción de insulina explicarían los niveles más bajos de $\mathrm{N}-1$ en el grupo con diabetes ${ }^{42}$. Por contra, otro estudio con 220 voluntarios chinos divididos en tres grupos (DM2 de recién diagnóstico, IGT y controles), observó que los niveles de $\mathrm{N}-1$ en plasma fueron mayores en los grupos con DM2 e IGT, comparados con los controles, pero no se presentaron diferencias entre los grupos de DM2 e IGT ${ }^{43}$ (Tabla 1).

\section{CONCLUSIONES}

La N-1 es una hormona anorexigénica de reciente descubrimiento que se expresa y secreta a nivel central y periférico. A nivel gastrointestinal, la $\mathrm{N}-1$ disminuye la ingesta de alimentos al inhibir el vaciamiento y reducir la motilidad. También se considera una hormona antihiperglucémica que estimula la secreción de insulina y mejora la sensibilidad a la insulina periférica. Algunos hallazgos son contradictorios, por lo que se sigue investigando cómo funciona la $\mathrm{N}-1$ en el organismo.

\section{BIBLIOGRAFÍA}

1. Ding S, Qu W, Dang S, Xie X, Xu J, Wang Y, et al. Serum nesfatin-1 is reduced in type 2 diabetes mellitus patients with peripheral arterial disease. Med Sci Monit. 2015;21:987-91.

2. Oh-I S, Shimizu H, Satoh T, Okada S, Adachi S, Inoue K, et al. Identification of nesfatin-1 as a satiety molecule in the hypothalamus. Nature. 2006;443(7112):709-12.

3. Mirzaei K, Hossein-nezhad A, Keshavarz S, Koohdani F, Eshraghian M, Saboor-Yaraghi A, et al. Association of nesfatin-1 level with body composi- 
tion, dietary intake and resting metabolic rate in obese and morbid obese subjects. Diabetes Metab. 2014;9(4):292-8.

4. Hofmann T, Elbelt U, Ahnis A, Rose M, Klapp BF, Stengel A. Sex-specific regulation of NUCB2/nesfatin-1: Differential implication in anxiety in obese men and women. Psychoneuroendocrinology. 2015;60:130-7.

5. Stengel A, Mori M, Taché $Y$. The role of nesfatin-1 in the regulation of food intake and body weight: recent developments and future endeavors. Obes Rev. 2013;14(11):859-70.

6. Ayada C, Toru U, Korkut Y. Nesfatin-1 and its effects on different systems. Hippokratia. 2015;19(1):4-10.

7. Hofmann $T$, Webert $E$, Ahnis A, Obbarius A, Elbert U, Rose M et al. Alterations of circulating NUCB2/nesfatin-1 during short term therapeutic improvement of anxiety in obese inpatients. Psychoneuroendocrinology. 2017;79:107-15

8. Prinz P, Goebel-Stengel M, Teuffel P, Rose M, Klapp BF, Stengel A. Peripheral and central localization of the nesfatin-1 receptor using autoradiography in rats. Biochem Biophys Res Commun. 2016;470(3):1-24.

9. Hofmann T, Ahnis A, Elbelt U, Rose M, Klapp B, Stengel A. NUCB2/nesfatin-1 is associated with elevated levels of anxiety in anorexia nervosa. Plos One. 2015;10(7):e0132058.

10. Feijóo-Bandín S, Rodríguez-Penas D, García-Rúa V, Mosquera-Leal A, Otero MF Pereira E, et al. Nesfatin-1 in human and murine cardiomyocytes: Synthesis, secretion, and mobilization of GLUT-4. Endocrinology. 2013;154(12):4757-67.

11. Blanco AM, Bertucci JI, Delgado MJ, Valenciano Al, Unniappan S. Tissuespecific expression of ghrelinergic and NUCB2/nesfatin-1 systems in goldfish (Carassius auratus) is modulated by macronutrient composition of diets. Comp Biochem Physiol A Mol Integr Physiol. 2016;195:1-9.

12. Irannejad A, Ghajar A, Afarideh M, Khajeh E, Noshad S, Esteghamati S, et al. Association of peripheral nesfatin-1 with early stage diabetic nephropathy. Pathophysiology. 2016;24(1):17-22.

13. Celik A, Gurger M, Can C, Balin M, Gul E, Kobat MA, et al. The effect of nesfatin-1 levels on paroxismal supraventricular tachycardia. J Investig Med. 2013;61(5):852-5.

14. Prinz P, Teuffel P, Lembke V, Kobelt P, Goebel-Stengel M, Hofmann T, et al. Nesfatin-130-59 injected intracerebroventricularly differentially affects food intake microstructure in rats under normal weight and diet-induced obese conditions. Front Neurosci. 2015;9(422):1-12.

15. Cui H, Sohn J, Gautron L, Funahashi H, Williams KW, Elmquist JK, et al. Neuroanatomy of melanocortin-4 receptor pathway in the lateral hypothalamic area. J Comp Neurol. 2012;520(18):4168-83.

16. Finelli C, Martelli G, Rossano R, Padula MC, La Sala N, Sommella L, et al. Nesfatin-1: role as possible new anti-obesity treatment. EXCLI J. 2014:13:586-91.

17. Cao X, Liu XM, Zhou LH. Recent progress in research on the distribution and function of NUCB2/Nesfatin-1 in peripheral tissues. Endocr J. 2013;60(9):1021-7.

18. Shimizu H, Oh-I S, Hashimoto K, Nakata M, Yamamoto S, Yoshida N, et al. Pheripheral administration of nesfatin-1 reduces food intake in mice: the leptin-independent mechanism. Endocrinology. 2009;150(2):662-71.

19. Zhang Y, Lu JH, Zheng SY, Yan JH, Chen L, Liu X, et al. Serum levels of nesfatin-1 are increased in gestational diabetes mellitus. Gynecol Endocrinol. 2017;33(8):621-4

20. Bluher M. Adipokines-removing road blocks to obesity and diabetes therapy. Mol Metab. 2014;3(3):230-40.

21. Osaki A, Shimizu H. Peripheral administration of nesfatin-1 increases blood pressure in mice. Hypertens Res. 2014;37(2):185-6.

22. Ramanjaneya M, Chen J, Brown JE, Tripathi G, Hallschmid M, Patel S, et al. Identification of nesfatin-1 in human and murine adipose tissue: a novel depot-specific adipokine with increased levels in obesity. Endocrinology. 2010;161(7):3169-80.

23. St-Pierre DH, Martin J, Shimizu H, Tagaya Y, Tsuchiya T, Marceau S, et al. Association between nesfatin-1 levels and metabolic improvements in severely obese patients who undervent biliopancreatic derivation with duodenal switch. Peptides. 2016;86:6-12.
24. Dogan U, Bulbuller N, Cakir T, Habibi M, Mayir B, Koc U, et al. Nesfatin-1 hormone levels in morbidly obese patients after laparoscopic sleeve gastrectomy. Eur Rev Med Pharmacol Sci. 2016;20(6):1023-31.

25. Ozkan Y, Timurkan ES, Aydin S, Sahin I, Timurkan M, Citil C, et al. Acylated and desacylated ghrelin, preptin, leptin and nesfatin-1 peptide changes related to the body mass index. Int J Endocrinol. 2013;2013:236085.

26. Tan BK, Hallschmid M, Kern W, Lehnert H, Randeva HS. Decreased cerebrospinal fluid/plasma ratio of the novel satiety molecule, nesfatin-1/ NUCB2, in obese humans: evidence of Nesfatin-1/NUCB2 resistance and implications for obesity treatment. J Clin Endocrinol Metab. 2011;96(4): E669-73.

27. Saldanha JF, Carrero JJ, Lobo JC, Stockler-Pinto MB, Leal VO, Calixto A, et al. The newly identified anorexigenic adipokine nesfatin-1 in hemodialysis patients: Are there associations with food intake, body composition and inflammation? Regulatory Peptides. 2012;173(1-3):83-5

28. Anik A, Catli G, Abaci A, Kume T, Bober E. Fasting and postprandial levels of a novel anorexigenic peptide in childhood obesity. J Pediatr Endocr Met. 2014;27(7-8):623-8.

29. Ustabas KF, Vehapoglu A, Tolga OI, Terziogu S, Cesur Y, Dundaroz R. Correlation of brain neuropeptide (Nesfatin-1 and Orexin-A) concentrations with anthropometric and biochemical parameters in malnourished children. J Clin Res Pediatr Endocrinol. 2015;7(3):197-202.

30. Stengel A, Taché Y. Gastric peptides and their regulation of hunger and satiety. Curr Gastroenterol Rep. 2012;14(6):480-8.

31. Stengel A, Hofmann T, Goebel-Stengel M, Lembke V, Ahnis A, Elbelt U, et al. Ghrelin and NUCB2/nesfatin-1 are expressed in the same gastric cell and differentially correlated with body mass index in obese subjects. Histochem Cell Biol. 2013;139(6):909-18.

32. Xu L, Wang H, Gong Y, Pang M, Sun X, Guo F, et al. Nesfatin-1 regulates the lateral hypothalamic area melanin-concentrating hormone-responsive gastric distension-sensitive neurons and gastric function via arcuate nucleus innervation. Metabolism. 2017:67:14-25.

33. Kentish SJ, Li H, Frisby CL, Page AJ. Nesfatin-1 modulates murine gastric vagal afferent mechanosensitivity in a nutritional state dependent manner. Peptides. 2017:89:35-41.

34. Gao S, Guo F, Sun X, Zhang N, Gong Y, Xu L. The inhibitory effects of nesfatin-1 in ventromedial hypothalamus on gastric function and its regulation by nucleus accumbens. Front Physiol. 2017;7(634):1-12.

35. Ramesh N, Mortazavi S, Unniappan S. Nesfatin-1 stimulates cholecystokinin and suppresses peptide $Y Y$ expression and secretion in mice. Biochem Biophys Res Commun. 2016;472(1):201-8.

36. González R, Reingold BK, Xiaodong G, Gaidhu MP, Tsushima RG, Unniappan S. Nesfatin-1 exerts a direct, glucose-dependent insulinotropic action on mouse islet beta- and MIN6 cells. J Endocrinol. 2011;208(3):R9-16.

37. Dai R, Deng G, Sun Z, Liu Z, Qian Y, Han Y. Relation of serum and vitreous nesfatin-1 concentrations with diabetic retinopathy. J Clin Lab Anal. 2017;31(5)

38. Bergmann K, Kretowicz M, Manitius J, Sypniewska G. Gender differences in association of serum nesfatin-1 with selected metabolic risk factors in normoglycemic subjects: A preliminary study. J Diabetes. 2015;7(3):433-4.

39. Ahmadizad S, Avanzar AS, Ebrahim K, Avandi M, Ghasemikaram M. The effects of short-term high-intensity interval training vs. Moderate-intensity continuous training on plasma levels of nesfatin-1 and inflammatory markers. Horm Mol Biol Clin Invest. 2015;21(3):165-73.

40. Li Z, Gao L, Tang H, Yin Y, Xiang X, Li Y, et al. Peripheral effects of nesfatin-1 on glucose homeostasis. Plos One. 2013:8(8):1-10.

41. Su Y, Zhang J, Tang Y, Bi F, Liu JN. The novel function of nesfatin-1: Antihyperglycemia. Biochem Biophys Res Commun. 2010;391(1):1039-42.

42. Algul S, Oskan Y, Ozcelik 0 . Serum Nesfatin-1 levels in patients with different glucose tolerance levels. Physiol Res. 2016;65(6):979-85

43. Zhang Z, Li L, Yang M, Liu H, Boden G, Yang G. Increased plasma levels of Nesfatin-1 in patients with newly diagnosed type 2 diabetes mellitus. Exp Clin Endocrinol Diabetes. 2012;120:91-5. 